\title{
Monitoring of strain of in-service railway switch rails through field experimentation
} Andrew Cornish ${ }^{1,2}$, Roderick A Smith ${ }^{2}$, John Dear ${ }^{2}$

1. Network Rail, The Quadrant:MK, Elder Gate, Milton Keynes, Buckinghamshire, MK9 1EN

2. Imperial College London, South Kensington Campus, London, SW7 2AZ

\begin{abstract}
In the financial year 2009/10, the Great Britain (GB) rail infrastructure manager, Network Rail, spent $£ 32$ million on the failures within switches and crossings (S\&C). Approximately 53\% of those failures occurred within the switch panel. In addition, two major incidents in GB in recent years have highlighted a lack of understanding of the loads and vibrations experienced by, and the consequent rates of deterioration of, switch panels.

This paper describes work which has been undertaken to help improve understanding of in-service loads experienced by switch panels and their consequent deterioration rate. Field experimentation has been designed and installed on four sites around GB, with the same design of switch. The change in response to loading, and the rate of deterioration, of the switch panels at each site was monitored over time. The effect of the vehicles and the deterioration were analysed individually before a comparison between the four sites. The analysis from the strain gauge measurements showed that there was an increase in the variance and maximum strains generated on the stock rail with the switch closed compared to when the switch is open. Finite Element Analysis (FEA) was used to validate variation recorded by the strain gauges under similar loads.
\end{abstract}

\section{Keywords: Switches and crossings, field experimentation, strain, failure statistics}

\section{Introduction}

The aim of this research was to understand the change in material response through site observation and monitoring over time. The infrastructure manager can use this information to monitor the various stages of the asset life cycle. Greater understanding of the assets response to loading types and ground conditions are necessary for the prediction of deterioration trends that can be used to increase efficiency of maintenance and renewal practices. This research was initiated from the lack of knowledge of forces being imparted to the S\&C from vehicles, which was highlighted by the Office of Rail Regulation (ORR) and Rail Accident and Investigation Branch (RAIB) investigation into the Grayrigg derailment [1]. Section 414 of the RAIB report states that: “... Network Rail's incomplete understanding of the design and performance of $S \& C$, and it's inspection and maintenance requirements, was an underlying factor in the accident at Grayrigg" [1]. This shows that the forces and the deterioration of the switch and crossing unit were not well understood by the industry.

Switches and crossings (S\&C) are complex assets; with moveable sections of rail manoeuvred by points operating equipment (POE), and longitudinally profiled switch rails to provide the ability for train running on various routes in a safe manner. On the GB railway infrastructure, there are over 20,000 sets of S\&C. In addition to this quantity, the complexity arises from over 200 different varieties of S\&C on the GB infrastructure through changes in the design, manufacturing and installation variables. S\&Cs have historically taken $24 \%$ of the maintenance budget and $23 \%$ of the renewal against $5 \%$ of the track miles [2].

A typical S\&C unit has an expected life of 25 to 60 years through regular operations, with the period of renewal dependant on the speed and tonnage of trains passing over the unit. This duration has been determined through historic renewal data and excludes any defects that may shorten life, such as poor geometry and manufacturing irregularities. For routes with high 
speeds, above 128kph, or tonnage above 25EMGTPA (Equivalent Million Gross Tonnes Per Annum), the expected life is 25 years. These types of turnouts have $2.1 \%$ of the total GB S\&C population currently. An S\&C unit is shown in Figure 1 [3]. The typical life increases incrementally up to 60 years for routes with speeds below 40kph and 6EMGTPA, which make up $23 \%$ of S\&Cs.

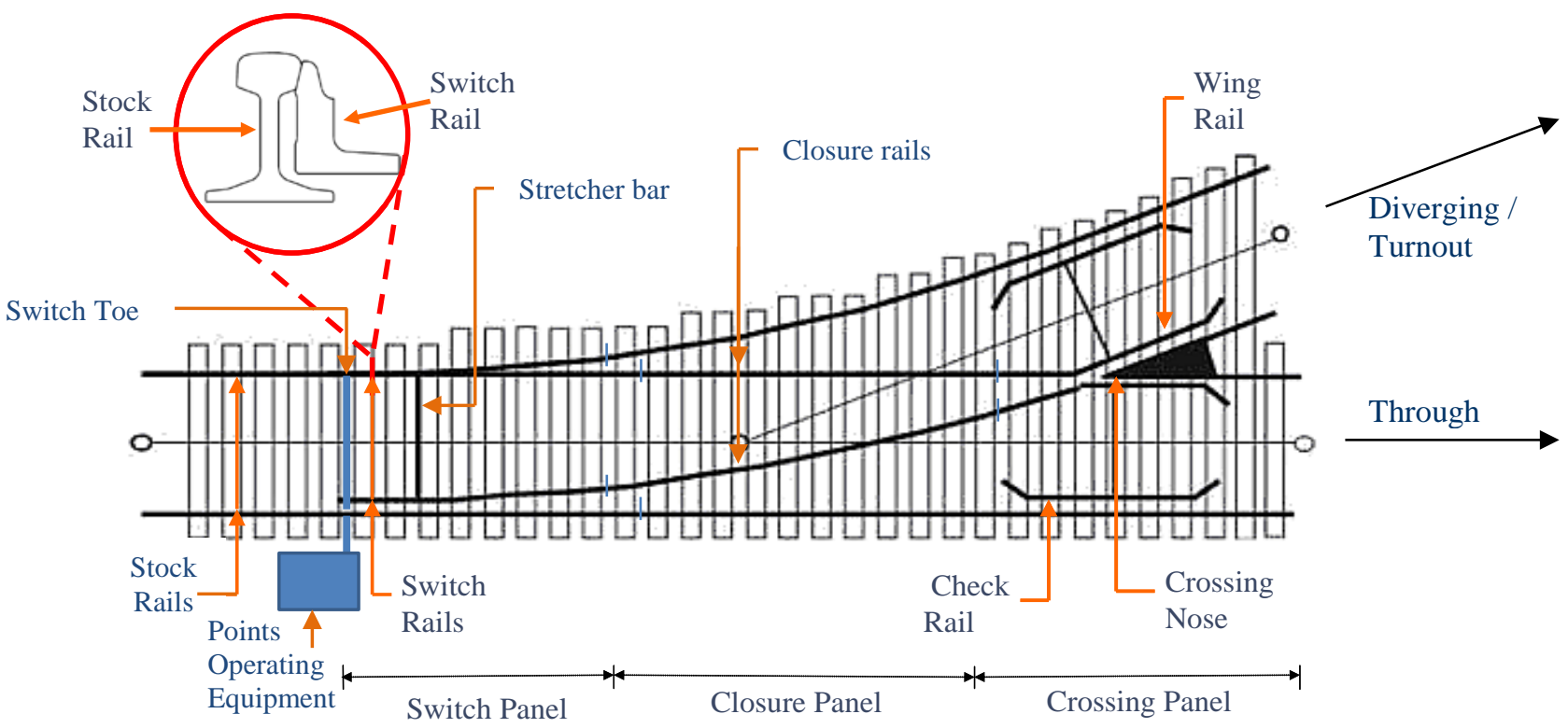

Figure 1: General switch and crossing unit [3] with a shallow depth switch profile

The moveable section of the switch was originally designed by Charles Fox in 1832 [4], with the design including profiled section of rail, and the moveable components. These design principals are still used in current S\&C. Before the design by Fox, sliding rails were used to allow for transferring between routes.

British Rail Research (BRR) completed research focused on the instrumentation of crossings due to the cost of manufacture and installation and thus switches in GB have not been instrumented. BRR instrumented crossings in the 1970s and 1980s to compare strain found from loading due to variations in designs, manufacturing companies and various vehicles impact [5-8]. 39 gauges were used by Boutle [7] in 1978 to compare bending strains of two crossings, with Groom [8] studying the effects of speed restrictions on the strain in crossings, as discussed in Infrastructure based experimentation.

More recently, the modelling of S\&Cs has been completed focusing on the contact conditions between the wheel and the rail and the effect of the variable wheel and rail profiles [9-11].

\section{The case for using field experimentation}

Experimentation can be split into two areas: laboratory and field testing. Laboratory testing has the advantage of completing large numbers of cycles within a controlled environment, but would be complex and costly for a whole switch panel, with large amounts of space and complicated rigs needed. For switch rails, field experimentation is the best way to monitor the change in strain over the life cycle of the asset as it monitors in-service vehicles including the effect related to rolling contact.

Field experimentation can be separated into two sections; vehicle based and infrastructure based. 


\section{Vehicle based experimentation}

Vehicle based instrumentation allows forces and loading variations over the whole asset to be monitored from the vehicle perspective, examples include loaded flatbeds for vertical forces [5] and more recently through instrumented wheelsets, such as Kassa [9]. This allows for measuring many different types of S\&C under known and controlled loading. This output includes the vehicles response to a change in the contact conditions and the discontinuities in the running path. The advantage of this would be for a single passage over an asset to collect data at all locations along the S\&C unit or to compare the different types of infrastructure assets. Disadvantages include it being very complicated to align multiple runs to monitor deterioration, the measurements are only at the location of the contact between the wheel and rail so highly susceptible to wheel profile on the measurement vehicle and being aligned to the majority of the traffic that traverses the unit. The monitoring of a single vehicle would mean that a comparison between freight and passenger would be difficult to monitor.

\section{Infrastructure based experimentation}

Infrastructure based instrumentation allows for a single asset to be monitored over a period of time and under multiple vehicles and vehicle types. This allows for monitoring the effects of a variety of traffic and a time history of forces against strain as the vehicle approaches. Six test sites were set up in the INNOTRACK European project, with varying level of instrumentation and location, with sites on Deutsche Bahn (Germany infrastructure) and Trafikverket (Swedish infrastructure). An example of the research completed using strain gauges on a crossing was on German infrastructure in Haste [12], where a crossing was installed with no switch panel. Nicklisch et al. [12] were able to calculate the vertical wheel / rail contact forces for a single locomotive, with a maximum wheel-load of $172 \mathrm{kN}$. The force measured was for a 1:12 crossing. This crossing angle is not found in GB and there was no research completed on the strain within the switch in these tests. Groom [8] states that the variation in strain due to speed through crossings is minimal. In his research in 1973, strain gauges were placed on speed restricted sites of the crossing on East Coast Main Line. Type 4 coaches were monitored over the site with speeds ranging from $60 \mathrm{kph}$ to $125 \mathrm{kph}$, which gave strains of between $55 \mu \varepsilon$ and $400 \mu \varepsilon$. Groom states that the higher strain values are exacerbated due to poor ground conditions.

\section{Failure Analysis}

Table 1 shows the failure statistics between 2006 and 2013, and their correlation to the S\&C failure numbers to show the percentage of failures that are seen within S\&C units. These failure minutes include all delays of the S\&C, including POE, and show a reduced delay annually for S\&C in the 5 years of the study.

Table 1: The Network Rail delay minutes statistics [2]

Financial Years

\begin{tabular}{|c|c|c|c|c|c|c|c|}
\hline & $2006 / 07$ & $2007 / 8$ & 2008/09 & $2009 / 10$ & $2010 / 11$ & $2011 / 12$ & 2012/13 \\
\hline $\begin{array}{c}\text { Network delay } \\
\text { minutes (million) }\end{array}$ & 10.53 & 9.5 & 8.84 & 8.18 & 8.95 & 8.34 & 8.80 \\
\hline S\&C delay minutes & & & 744,297 & 657,883 & 643,408 & 593,679 & 575,870 \\
\hline $\begin{array}{l}\text { S\&C delay } \\
\text { percentage }\end{array}$ & & & 8.42 & 8.04 & 7.19 & 7.12 & 6.54 \\
\hline
\end{tabular}


The delay minutes are still larger than the $5 \%$ of the infrastructure that the S\&C comprise.

The failure analysis in this research used three Network Rail databases. These are:

- GEOGIS: store of asset information, such as location, type of asset and age. The benefits include being the main store of infrastructure asset knowledge for GB. The issue is that the database has been found to be c. 90\% accurate [13], with errors historically occurring from inaccuracies through updates.

- Fault Management System (FMS): FMS manages and reports the failures on infrastructure assets. The database shows and explains the details of failures from the infrastructure perspective. FMS is currently aligned with GEOGIS for location and asset information, with the inaccuracies from GEOGIS also pertinent within FMS.

- Train Running System (TRUST): TRUST records all the details of running vehicle and aligns with the schedule. If a vehicle is late into a station, TRUST generates a number that can have the cumulative delays and associated costs attributed. TRUST can be aligned to FMS to give a complete understanding of the delays to vehicles and the cause.

These three databases give information on the type of switch unit that has failed, the reason behind the failure and the costs that are associated to the failure through delays. Additional details on some of the process of events to resolve the failure were also included, but the quality of the recording varies depending on the description and knowledge of the data entry. The data that was used in this research was from January 2009 to May 2012 for all S\&C failures, and the associated delay cost for the failures.

\section{Component failure}

Each S\&C unit contains many individual components: e.g. rail; sleepers; slide baseplates; clips. To ensure that the instrumentation is in the correct location to give the most beneficial outputs, the delay costs attributed to the various components are identified from the TRUST database. The justification to understand the failures of the various components within the S\&C unit is to ensure that the instrumentation outputs are highly pertinent to the failures within S\&C units.

The justification for this research focusing on switches rather than the entire unit was due to a large percentage of failures within the switch panel. Figure 2 shows the component failures within the S\&C unit and reveals that the switch blade was the most likely to fail, with $53 \%$ of components of S\&C railway asset (e.g. rail, ballast) failures. The failures are for the track based assets only, and points operating equipment statistics were removed as these were not considered in the research. This trend was seen throughout the various lengths of S\&C units. This trend was due to the moveable component of the rail emphasising stress raisers which generates a higher dynamic loading from the transfer from the switch rail to the stock rail. Figure 2 shows the switch rail generates a high cumulative delay due to compromising the safety of the railway and the necessity to place a speed restriction or stop vehicles from running until the rectification is completed. 


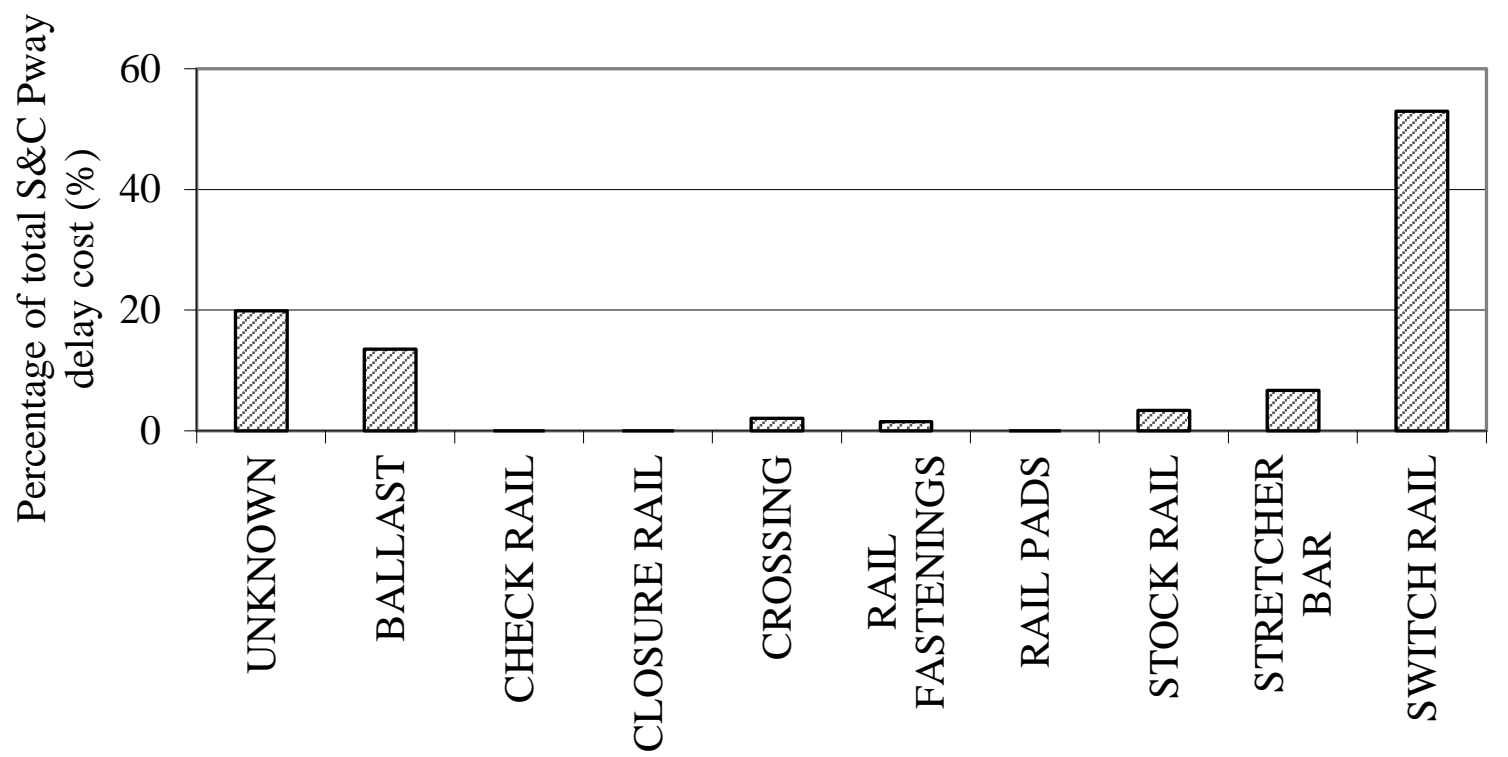

Figure 2: The percentage of 'permanent way' S\&C component delay cost

It has been found that the switch is the key component within switches and crossings by studying the total delay costs that was imposed on Network Rail from January 2009 to May 2012.

Table 2 shows some of the design characteristics for the shallow depth, vertical switch and crossing units from the Network Rail Track Design Handbook, NR/L2/TRK/2049 [14]. Figure 3 shows a shallow depth switch, which have a fully profiled stock rail and a manufactured reduced height switch blade that is positioned above the stock rail foot on a baseplate.

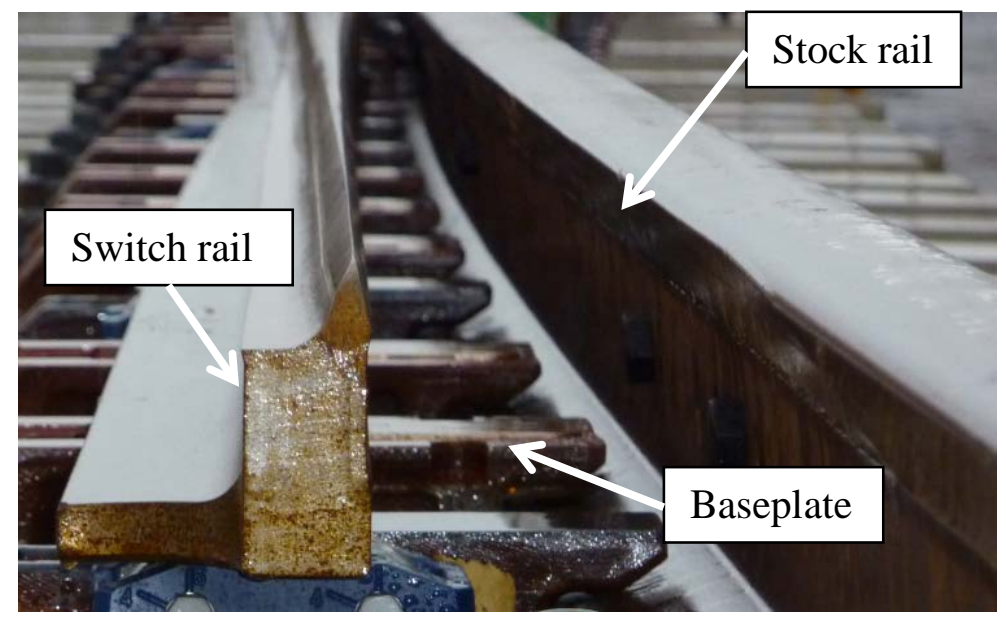

Figure 3: A shallow depth switch rail

$\mathrm{G}+$ switch length category has combined longer assets with a small population to simplify some of the analysis, whilst still representing the higher speed aspect of the S\&C populations. The design characteristics (24.4m turnout length; $1264 \mathrm{~m}$ switch radius) are for the $\mathrm{G}$ length design as that is the smallest design that is included in the $\mathrm{G}+$ classification. 
Table 2: The designs of the switches and crossings for CEN56 vertical (V) design [14]

\begin{tabular}{ccccc}
\hline $\begin{array}{c}\text { Switch } \\
\text { Classification }\end{array}$ & $\begin{array}{c}\text { Turnout length, } \\
(\mathrm{m})\end{array}$ & $\begin{array}{c}\text { Switch radius, } \\
(\mathrm{m})\end{array}$ & $\begin{array}{c}\text { Allowable } \\
\text { speed }(\mathrm{kph})\end{array}$ & $\begin{array}{c}\text { Population } \\
(\%)\end{array}$ \\
\hline A & 7.3 & 141.1 & 32 & 1.5 \\
B & 8.7 & 184.0 & 32 & 15.3 \\
C & 11.9 & 245.8 & 40 & 48.2 \\
D & 12.4 & 332.7 & 48 & 16.1 \\
E & 17.3 & 645.1 & 64 & 12.5 \\
F & 20.8 & 980.9 & 80 & 3.6 \\
G+ & 24.4 & 1264 & $96+$ & 2.8 \\
\hline
\end{tabular}

\section{Switch and crossing failure mechanism}

With the information of the design of S\&C and the failure statistics, it was possible to understand which failure mechanism occurred within the various design and length of switch.

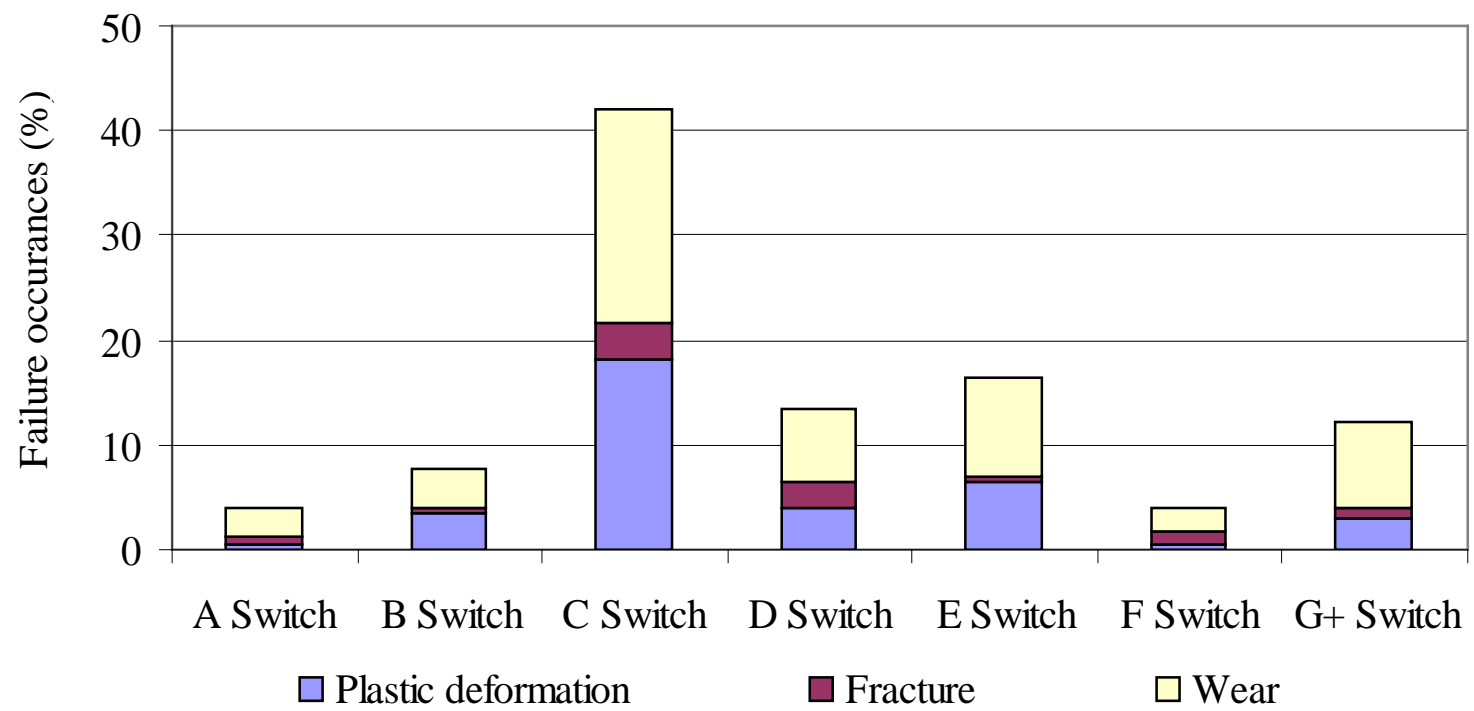

Figure 4: Percentage of total permanent way failure occurrences against switch length

Once the failure has been discovered, it is necessary to rectify the issues that have been raised to ensure that the railway is operational and safe. The common failure modes from the statistics have been grouped into three 'failure classifications': Plastic deformation; Fracture; and Wear. RCF is not included in the failure mode classification as the statistics show that less than $1 \%$ of delay costs were caused by RCF within S\&C.

The rectifications are the process to fix the issue that caused the delay. Combining a rectification cost with the delay costs gave the instrumentation an overall cost relevance, or failure cost. The costs included materials and labour to complete the rectification. When there is more than one rectification for a problem; i.e. Fracture can be a replacement unit or replacement weld, or weld repair; a percentage of each rectification costs were applied, using information within the databases on the rectification. 
From using the failure classifications, the associated switch lengths were investigated to see the variation of the damage mechanisms within classifications. Figure 4 shows the percentage of failures for each of the switch lengths and the accumulation of the failure mechanisms against the total number of failures within S\&C rail. It shows that the $C$ length switch has the largest percentage of failures over the network, which is due to the high population of the $\mathrm{C}$ length switch (48\%).

Plastic deformation (38.6\%) and worn (39.6\%) classifications are the two largest in failure costs, with the occurrences of fracture less often (21\%), but with greater costs for rectification. Figure 5 shows the total costs of the failures, including the rectification costs.

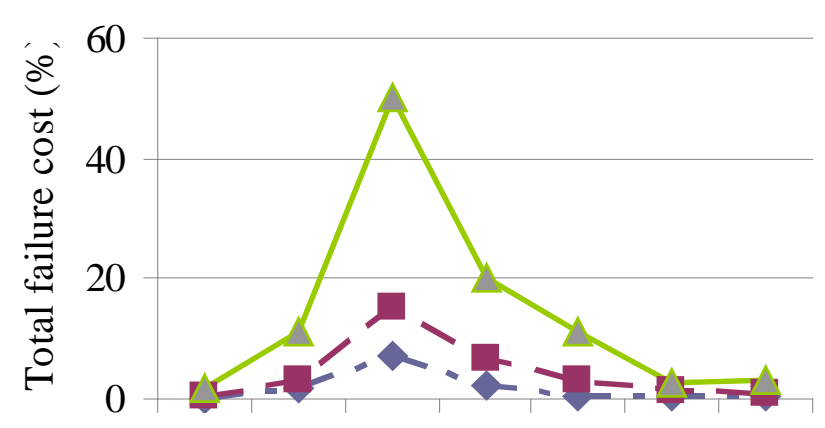

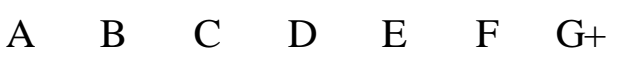
$->$ - Plastic deformation $-\square-$ Fractured Worn

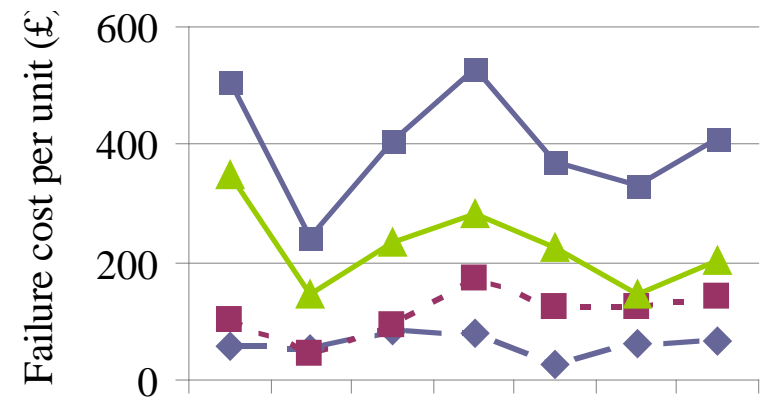

$\begin{array}{lllllll}\text { A } & \text { B } & \text { C } & \text { D } & \text { E } & \text { F } & G^{+}\end{array}$
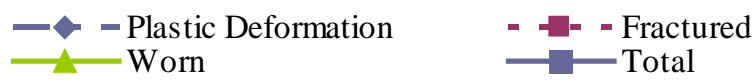

(b)

(a)

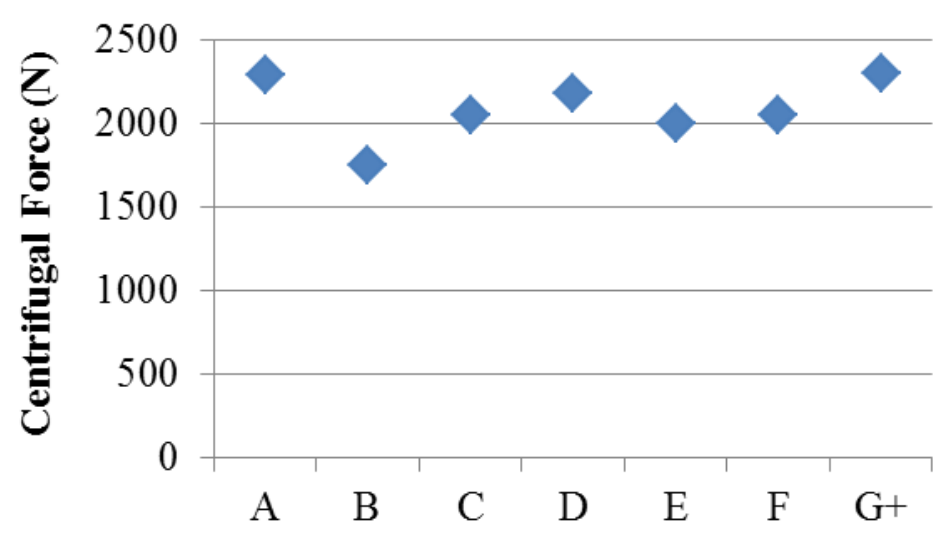

(c)

Figure 5: The costs of failures, (a) The percentage of total failure costs against switch length, (b) the failure cost per unit for the various failure modes against switch length and (c) the centrifugal force per switch length

From Figure 5(a) it can be seen that the switch length $C$ had the highest percentage of total failures costs. This was due to the large population of the $C$ length switch. In general, the higher speed switches tend to see more wear damage due to the dynamic forces removing the top layers of the material. The A length switch tend to stay in service for longer durations [3] due to their lower track classification which means that the unit is replaced due to excessive wear. When normalising the cost against the number of S\&C units, Figure 5 (b) shows the high speed (G+ Switch) and the low speed (A Switch) had higher costs / unit along with the mid speed (D Switch). Whilst the A length and G length switches had a higher failure cost / unit, the population is small in comparison. 
The lateral forces applied to the rail through the turnout route are a result of the change in rolling radius of the wheel on the rail. These forces are a result of the lateral offset of the contact due to the centrifugal forces applied on the vehicle and the relative velocity between the wheel and the rail [15] as they traverse the curve. Centrifugal force is calculated through equation

$$
F_{c}=\frac{W V^{2}}{g R}
$$

Where $\mathrm{F}_{\mathrm{c}}$ is the centrifugal force, $W$ is the weight of a vehicle, assumed $40 \mathrm{t}$, which is similar to a Mark 4 coach that runs on the East Coast Mainline, $V$ is the maximum allowable turnout speed and $R$ is the radius of the turnout.

Using the maximum speed of the turnout route, and assuming constant radius curves, the centrifugal forces have been calculated and shown in Figure 5 (c). The largest theoretical values for centrifugal forces are for the extreme lengths, the A length and $G$ length switches. After these extremes, the D length switch experiences the next highest centrifugal force. These higher centrifugal forces result in larger forces into the infrastructure and can manifest in increased likelihood of failure mechanisms, such as wear and RCF.

The mid-speed switch, D length, has the highest overall failure cost per unit, followed by the short, A length switch. The maximum failure mode is the worn classification, but the D length switch also has the highest figure for fractured rail. The highest cost per unit for the D switch is due to the large cumulative effect of the increased number in fractured rails.

The D length switch is the most significant for the first phase of instrumentation, excluding the extremes. This is due to $28 \%$ of failure costs and $£ 520 /$ unit, whilst having the second largest population of S\&C, $16 \%$.

In the financial year 2012/13, a total of 1500 failures were caused by the S\&C components, causing a delay to the network of over 115,000 minutes. This equates to a cost of over $£ 3$ million to the infrastructure company increasing to $£ 32$ million for all delays within S\&C, which includes POE. Failure statistics were analysed to understand the type of failure that occurs within the current design to ensure the instrumentation is positioned in the pertinent locations. S\&C are highly complex assets, which have many components with a 5\% track length against the total track distance of infrastructure. S\&Cs have design classifications to generate eight allowable speeds and infrastructure constraints. The statistical analysis was completed to focus the instrumentation parameters and understand the current in-service degradation of S\&C.

\section{Design of Experimentation}

Design of Experimentation (DOE) is a statistical tool that can allow for many designs to be monitored and analysed through a few representative units. Field experimentation is necessary to measure large quantities of in-service loading data that would otherwise be unobtainable due to reasons including complex contact between the wheel and the rail and limited space. Table 3 outlines the four sites within a DOE structure for the experimentation. A DOE procedure was set up in line with the results gained from the failures statistics [16] showing that the D length switch (13m switch rail length, 370m turnout radius [14]) has high industrial 
relevance due to significant delays, failure costs and large population. SC1 has been classified as the reference site and has been instrumented since installation to gain the initial wear rate phase.

Table 3: The design of experiments completed through the failure statistics and parameter understanding

\begin{tabular}{ccccccc}
\hline Unit Name & $\begin{array}{c}\text { Switch } \\
\text { Design }\end{array}$ & $\begin{array}{c}\text { Rail } \\
\text { Inclination }\end{array}$ & $\begin{array}{c}\text { Line } \\
\text { Speed }\end{array}$ & $\begin{array}{c}\text { Track } \\
\text { Quality }\end{array}$ & $\begin{array}{c}\text { Traffic } \\
\text { Type }\end{array}$ & $\begin{array}{c}\text { Annual } \\
\text { Tonnage }\end{array}$ \\
\hline SC1 & D & Vertical & V1 & Good & Mixed & T1 \\
SC2 & D & Vertical & V2 & Poor & Mixed & T3 \\
SC3 & D & Vertical & V3 & Satisfactory & Mixed & T1 \\
SC4 & D & Inclined & V3 & Good & Mixed & T2 \\
\hline
\end{tabular}

Where :
$\mathrm{V} 1=0-127 \mathrm{kph}$
$\mathrm{T} 1=0-7 \mathrm{MGTPA}$
$\mathrm{V} 2=128-160 \mathrm{kph}$
$\mathrm{T} 2=7.1-12 \mathrm{MGTPA}$
$\mathrm{V} 3=161+\mathrm{kph}$
$\mathrm{T} 3=12+\mathrm{MGTPA}$

\section{Block design parameters for Design of Experiments}

A block design is a DOE technique that ensures that pertinent parameters are covered for instrumentation from a statistical perspective. The block design was set up to allow for multiple parameters to be compared over the duration of the project. The subsequent sections explain the parameters, the various scalable levels, and the effect levels of the parameters were expected to have on the results from the various instrumentation sites. The five variable parameters and levels are;

- Rail inclination: vertical or inclined - Rail inclination changes the contact location as the wheel and rails contact moves with the inclined rail due to the crown design and the wheel profile being more conformal. This could change the deterioration rates that are seen through S\&C.

- Line speed: V1 (lowest) to V3 (highest) - The speed of the through route of the S\&C unit can make changes to the location of contact and the location of the transition.

- Track quality - Track geometry is a complex parameter to monitor and keep consistent. The track quality influences the behaviour of the vehicles and thus the forces within the contact patch. Three quantifications have been created, but the track quality will change throughout the experimentation.

- Traffic Type: Passenger, Freight or Mixed - Zwanenburg [3] commented that the higher the freight percentage, the lower the chance of failure as the freight routes tend to be used less, albeit by larger axle loads. 
- Annual tonnage: T1 (lowest) to T3 (highest) - The larger the annual tonnage, the more likely the asset will fail due to the loading and material removal.

\section{Method of instrumentation}

Monitoring of the S\&C behaviour and the change in response from loads was achieved through using strain gauges. Strain gauges were used as they have the ability to be attached to the rail to continuously generate data which can be monitored and analysed. The strain gauges were installed on the neutral axis (NA) to ensure that the bending strains were kept to a minimum and in the centre of sleeper spacing to remove the influence of the sleeper stiffness. As the vehicle passed over the gauges, the shear strain was recorded, without the influence of the structure bending. This allows for a greater and clearer understanding of the response of the material to the loading. The disadvantages include the installation on the NA is highly complicated, with the distance $82.26 \mathrm{~mm}$ from the base [17]. The influence of the bending strain increases as the gauge moves further from the NA, but has been determined through Finite Element Modelling [18] not to be a significant factor in the error levels, within vertical installation tolerances.

32 gauges were installed on the reference site (SC1), 16 gauges on non-reference (SC2-4) of which the following locations have been analysed within this paper:

- $1.2 \mathrm{~m}$ from switch tip as this monitors the loading on the switch tip and the moveable section of the rail

- $4.5 \mathrm{~m}$ from switch tip on reference site (on SC1) as this is within the influence of the transfer of wheels from one rail to another. This was not deemed to be installed on the non-reference sites as the tips were thought to be more significant.

- $8 \mathrm{~m}$ from switch tips to include some of the reduced web section to monitor the effect of lateral movement on the material response.

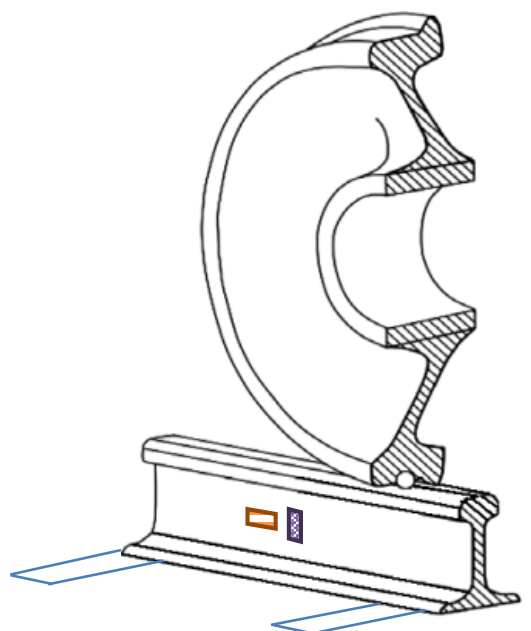

(a)

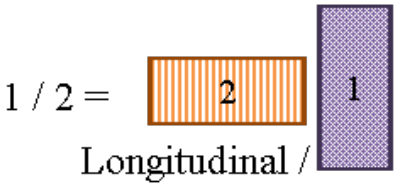

Axial
Vertical /

Transverse

Figure 6: The layout of strain gauges in the sleeper centre at (a) the neutral axis and (b) the names of the directions of the gauges, image from [18]

\section{Instrumentation Results}

The instrumentation was placed on S\&C units with varying time in service and different yearly loading due to the key parameters found in failure statistics and previous studies [16]. The variation in age of the S\&C units instrumented was to give a representation of the 
development of strain over a longer duration. The cumulative tonnage allowed monitoring of the change in strain due to the total vehicles that had traversed the units. Three of the four sites gave clear and useable data, with SC2 giving unreadable information due to presence of noise which could not be filtered out. This means the data gained from Site 2 was not used.

Figure 7 (a) - (e) shows the vertical RMS strains for all vehicles that passed over the site. The gauges in each figure are at the same location longitudinally along the sites. Standard deviation of the results gives an indication of the range and uncertainty of the loading from the vehicles. The standard deviation of the population was calculated for the various strain gauge locations to give a comparison of the strain ranges from cyclic loading, with variances in the contact and vertical loading.

When comparing the open and closed switch rails for newly installed units, standard deviation of the strain values show fairly similar values, with 11.7 for the open switch (Figure 7 (a)), whilst the closed switch (Figure 7 (b)), has a standard deviation of 17.8. This shows that there is a small change in the contact estimated through the closed switch blade which is due to the transfer of the loading. After 125MGT (Million Gross Tonnes), the increase in the standard deviation on the open switch rail is to 30.4. As the shear strain is being monitored, with bending strain being reduced due to the location of the gauges, this increase is due to the deterioration of the system and transfer of forces in the contact of the rail. Figure 7 (b) shows a much larger increase in the standard deviation over 125MGT, with an SD of 68.3.This larger uncertainty rises from the variations in the transfer from the switch rail to the stock rail and the additional dynamic forces induced by the transferring of contact from the switch rail to the stock rail [19].

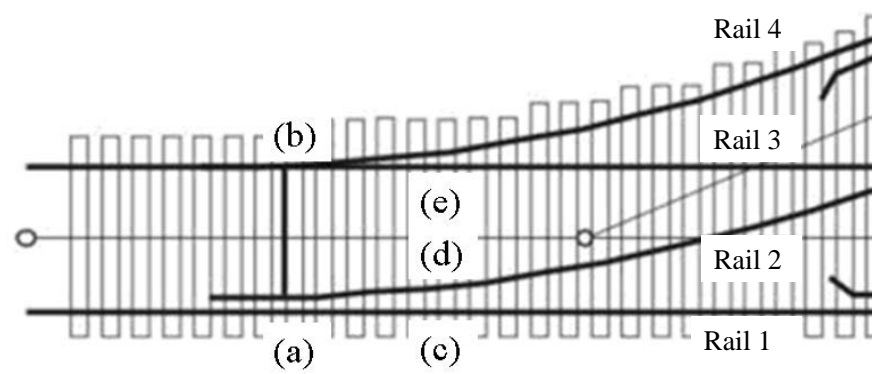

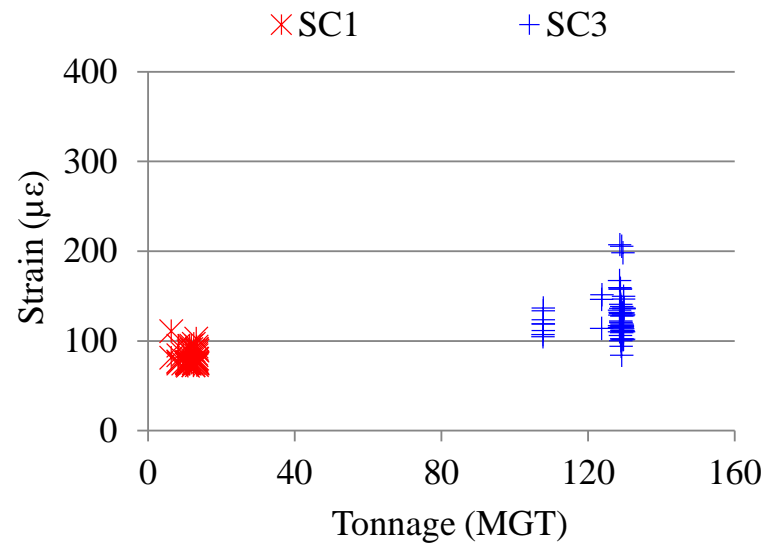

(a)

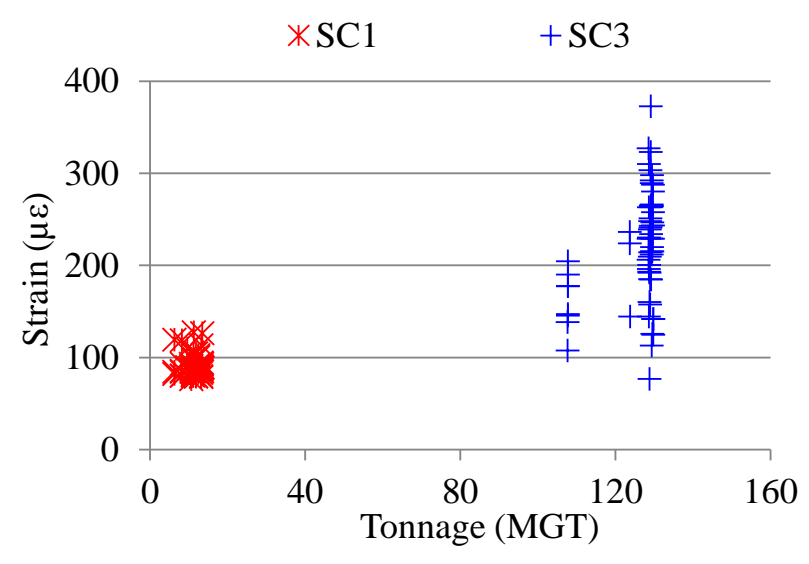

(b) 


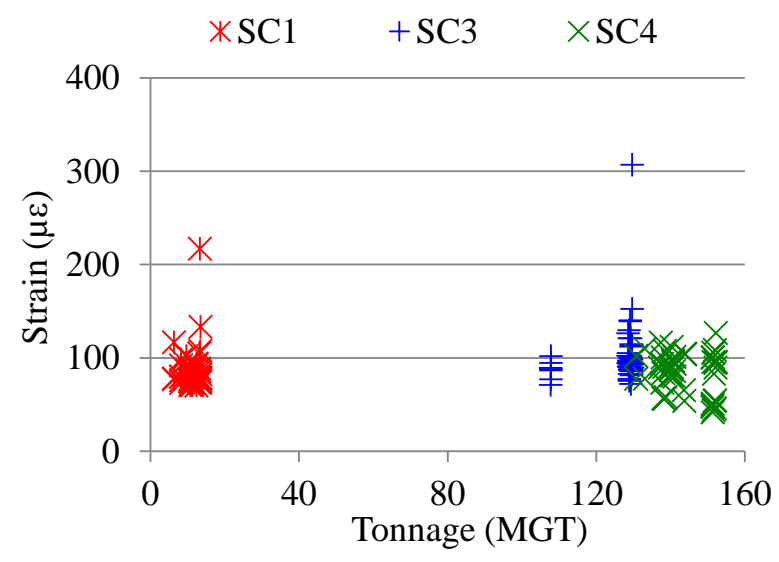

(c)

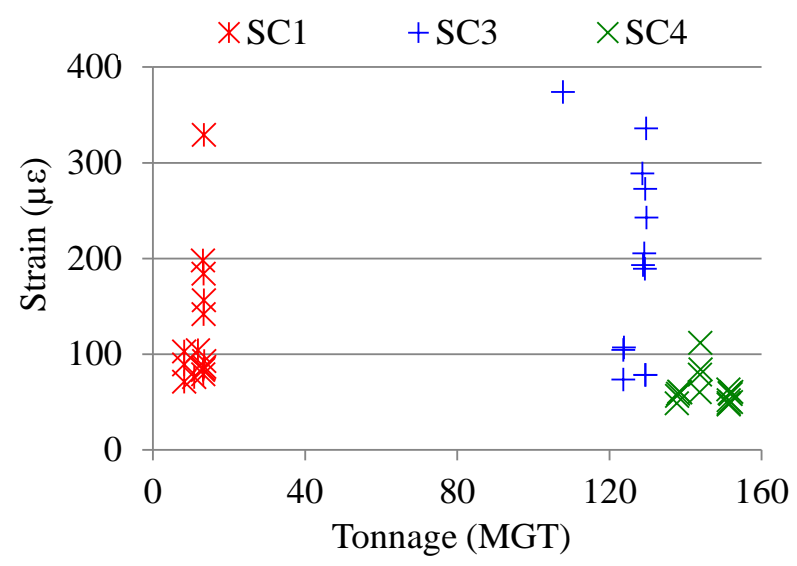

(d)

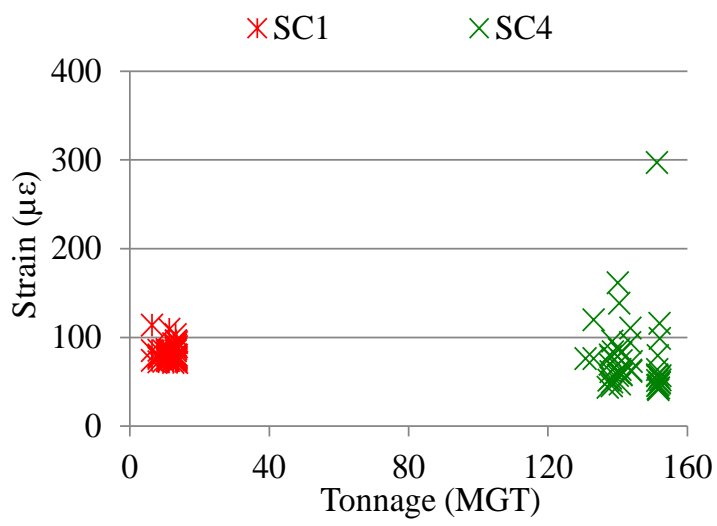

(e)

Figure 7: Root-mean squared (RMS) strains for the different sites against the total tonnage at the respective sites: (a) stock rail near switch tips; (b) the stock rail with closed switch near switch tips; (c) at $11 \mathrm{~m}$ on through route (d) at $11 \mathrm{~m}$ on turnout route; and (e) at $11 \mathrm{~m}$ on through route on the transferred stock rail.

Results from Rail 4 have not been shown due to the small (31 of 123 datasets) number of datasets that exceeded the $50 \mu \varepsilon$ RMS trigger. The traffic for Figure 7 (a)-(c) and (e) are only vehicles passing over the through route, with Figure 7 (d) showing the turnout routes. The change in strain is highest towards the switch tip, seen in Figure 7 (b). Figure 7 (d) shows larger strain, however, this is due to loading of heavier axles on the turnout route of which was populated by laden freight.

There has been a variation in the strain seen by similar vehicles at the same locations at the same tonnage. To understand this variation in response to similar loading, FEA modelling was carried out, which is shown in Figure 8.

\section{Validation}

Two conclusions from the results need to be explored to understand their impact. The two factors explored are:

1. The contact location is changing

2. The change in under rail stiffness over time.

To validate some of the reasons behind the variations in strain, a static Finite Element Analysis (FEA) model was created for a plain line section of rail. The purpose of this section is to give justification to the wide range of strain values that were recorded from similar 
vehicles. This was completed due to the output from the stock rail being monitored, and the additional complications from variations in geometry of the switch rail. A drawing of the model is shown in Figure 8, along with the outputs from the various models. A convergence was completed on the mesh, and a $2 \mathrm{~mm}$ hexahedral mesh through the gauge location was deemed most suitable due to the time to complete and $1.8 \%$ variance in output in line with smaller meshes. For the change in ground conditions over time, variations in the under rail stiffness were modelled, studying anomalies (with one sleeper variable stiffness), and deterioration (two or more sleeper variation). The steady state ground condition for a single rail is deemed to be $50 \mathrm{MN} / \mathrm{m}$ as that is the value used within industry as a steady state for normal railway was derived from the experimental studies [20]. Vampire ${ }^{\circledR}$ uses the value of $50 \mathrm{MN} / \mathrm{m}$ for the rail stiffness under contact conditions on a single side, i.e. the stiffness of the rail is included in the stiffness value. Industry experts [21-22] claim that the track stiffness varies from $25 \mathrm{MN} / \mathrm{m}$ (equivalent to a multiple (greater than 4) unsupported sleepers) to $200 \mathrm{MN} / \mathrm{m}$ (on very firm, contaminated ground) from experimental studies. The ground conditions for a single under rail stiffness in the first simulation, $\mathrm{k}_{\mathrm{f} 2}$, ranges from $10 \mathrm{MN} / \mathrm{m}$ to $100 \mathrm{MN} / \mathrm{m}$, and in the second scenario $\mathrm{k}_{\mathrm{f} 2}$ and $\mathrm{k}_{\mathrm{f} 3}$ are varied, with equal stiffness. The model enables a change of ground condition to be monitored, either with a track defect (single sleeper variation) or geometry irregularity (two sleepers). A load equivalent to 20.5 tonnes (1000MPa) was applied to the surface of the rail, which is equivalent to a Class 91, which are the locomotives on the East Coast Mainline High Speed Train.

Figure 8 shows the outputs from the model and the variation due to the change in lateral contact locations, and the two variable ground conditions shown above the plots. The change in the stiffness is shown along the $\mathrm{x}$-axis, and the $\mathrm{y}$-axis shows the strain that has been modelled and outputted from the location of the gauge.
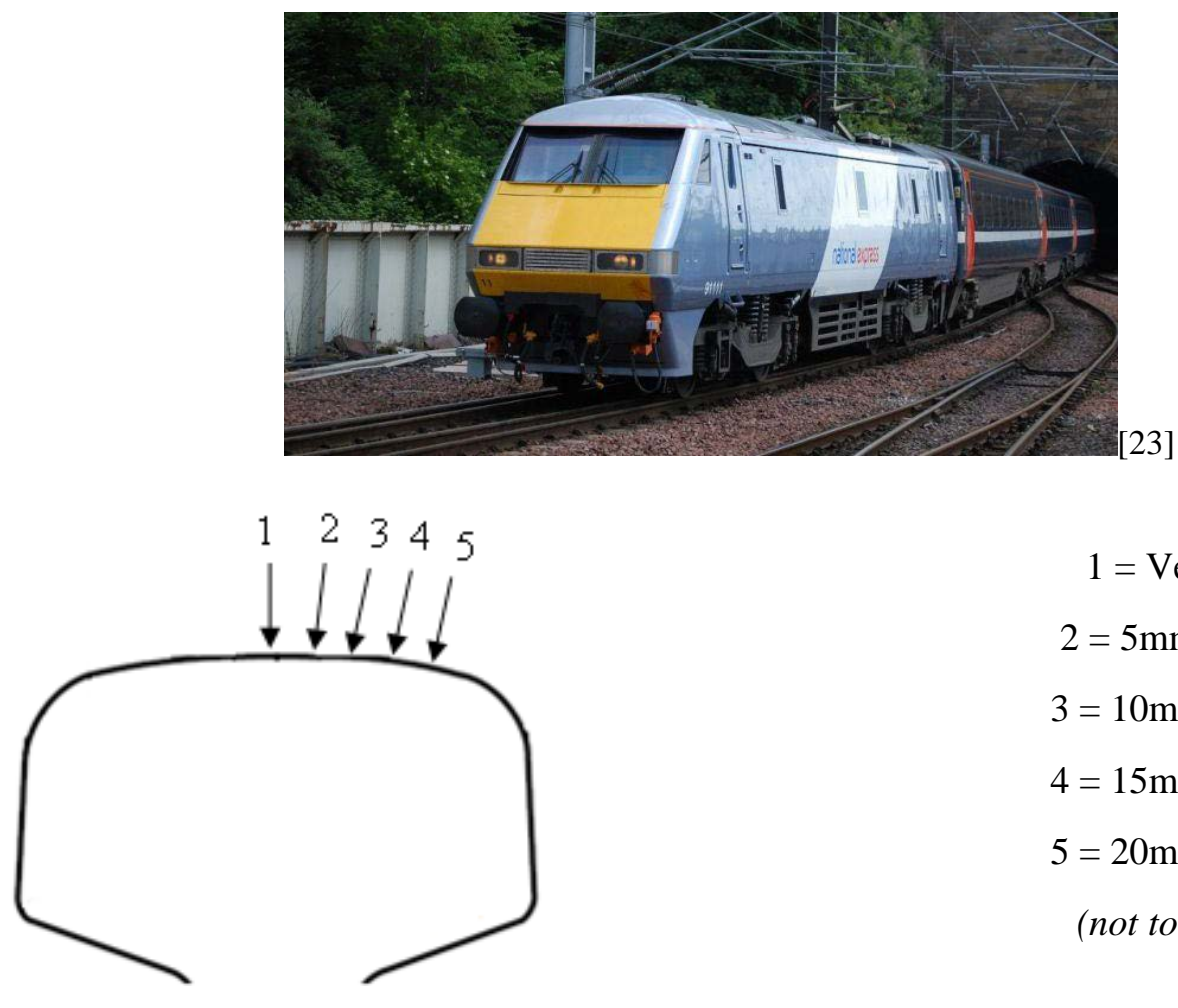

$$
\begin{gathered}
1=\text { Vertical } \\
2=5 \mathrm{~mm} \text { offset } \\
3=10 \mathrm{~mm} \text { offset } \\
4=15 \mathrm{~mm} \text { offset } \\
5=20 \mathrm{~mm} \text { offset } \\
\text { (not to scale) }
\end{gathered}
$$



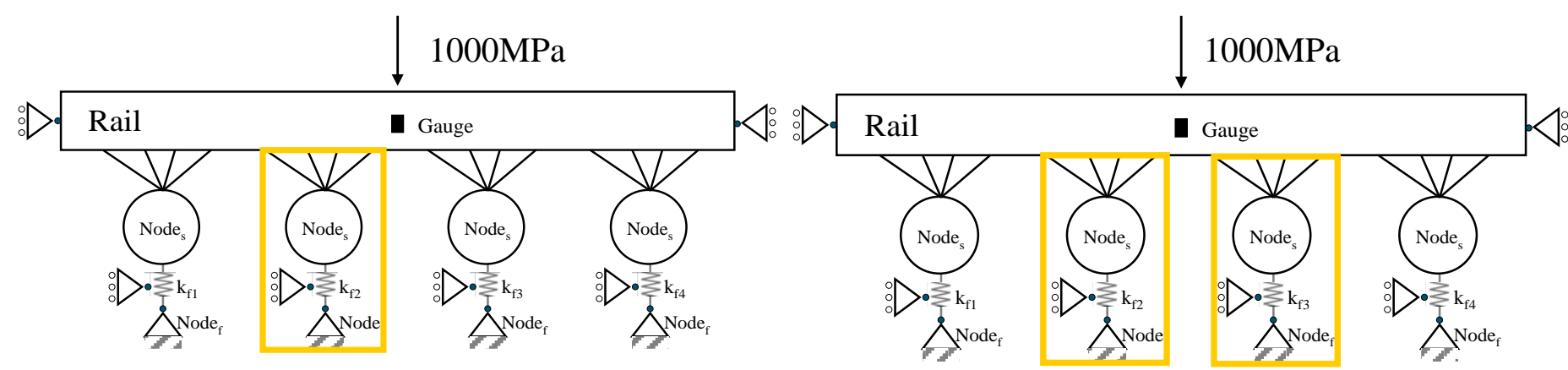

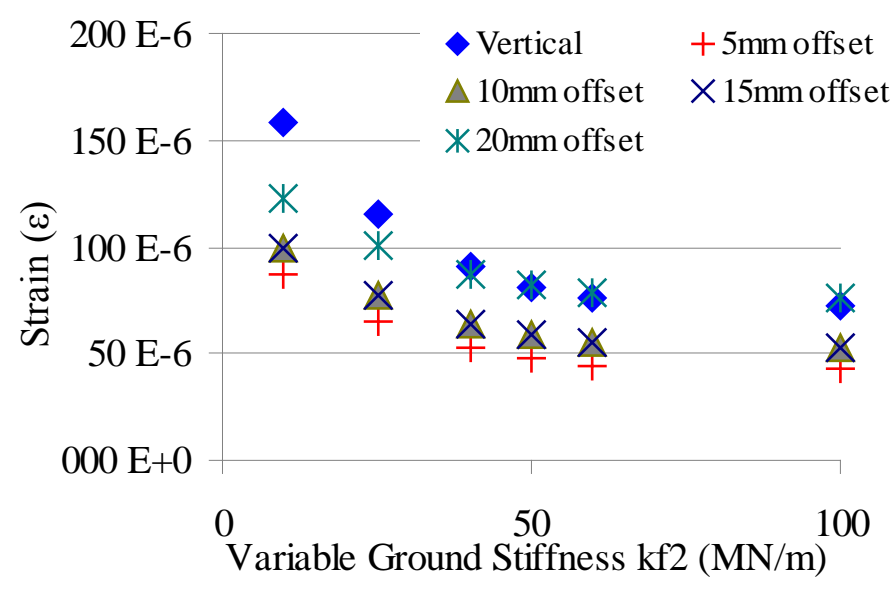

(a)

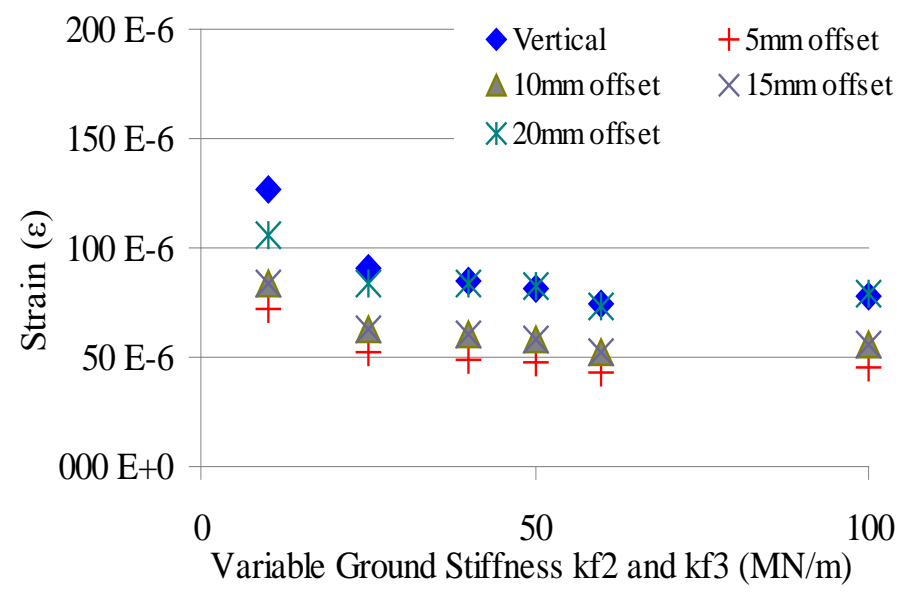

(b)

Figure 8: The strain generated at the gauge output location on a rail with varying vertical support stiffness and various contact conditions, with a) showing the single under-sleeper stiffness variation and b) showing two undersleeper stiffness variation

\section{Analysis and outcomes}

A $10^{\text {th }}$ order lowpass Butterworth filter was applied to the outputted data at $100 \mathrm{~Hz}$, as it was found that above that frequency was insignificant through an FFT analysis of the strain data [18]. $50 \mathrm{~Hz}$ was also removed through a passband filter due to the electromagnetic current (EMC), generating large amounts of noise. $10^{\text {th }}$ order Butterworth filters were found to give the cleanest removal of unwanted frequencies without distorting the surrounding data.

The model gave a range of strain at the standard stiffness $(50 \mathrm{MN} / \mathrm{m}$ or greater) of $40 \mu \varepsilon$, with $48 \mu \varepsilon$ at $5 \mathrm{~mm}$ to $90 \mu \varepsilon$ at vertical loading. The $5 \mathrm{~mm}$ offset is not uncommon on site; with the variation in the wheel profile due to wear often causing a shift in the contact location. In reality, this exact wheel profiles are not known for the sites, and thus the conclusion that this is the only reason for the variation in the strain is inconclusive.

An exponential decay relationship is shown for the strain against increasing ground stiffness, with stiffness greater than $50 \mathrm{MN} / \mathrm{m}$ showing a constant strain output. This shows that the ground conditions are also fundamental to the general condition of the rail; with a low ground conditions 2.5 times the strain values for a single sleeper and doubling the vertical loading for multiple sleeper variation. This is unlikely to be the cause of the variation of the single vehicles at a single site, but can be seen that the deterioration of the ground conditions have a large contribution to the strain over time. Whilst the conditions should not get to a stiffness of $10 \mathrm{MN} / \mathrm{m}$, the increase is still significant for the lower stiffness. It can also be seen that a 
single sleeper variation has a larger detrimental effect on the stiffness as the response to the loading has a larger deviation from the other sleepers, and thus the strain is increased.

Other contributing factors to the increase in the variable strain gauge outputs for the similar vehicles could be due to the dynamic effect of the loading as the vehicle travels over the gauge.

\section{Conclusions and future work}

Four sites, of which three sites were used, were selected and instrumented to understand the deterioration of switch through field experimentation. DOE from failure statistics showed that switch rail is highly pertinent as it sees the majority of the failures from the S\&C units. The failure costs have shown a variation of deterioration through plastic deformation and wear depending on the length of the switch. Field experimentation was installed on sites that were determined through DOE techniques, with six parameters for the experimentation examined: switch length; rail inclination; line speed; track quality; traffic type; and annual tonnage.

Strain gauges showed that there was a larger increase in the outputs for the stock rail with the closed switch blade due to the cumulative transfer from one rail (switch rail) to another (stock rail). A range in strain output of up to $90 \mu \varepsilon$ was recorded from the strain gauges for similar vehicles.

Validation of the variation from a single vehicle type has shown that a $5 \mathrm{~mm}$ variation in the contact position can increase the strain by c. $50 \mu \varepsilon$. This shows that a change in the lateral contact can have a large effect on the strain generated at the gauge. Validation was completed to study the effect on the general ground conditions to understand some effects of deterioration. A single sleeper variation (voiding) and a double variation (deterioration) were modelled and showed an increase of up to 2.5 times for a single sleeper and double for general deterioration. This shows that the quick change in ground conditions has a larger material response and thus variations over a small distance have greater significance in the deterioration than over larger distances.

The conclusions have been drawn from the information that has been collected. To solidify some of the conclusions, additional data is being collected and will be analysed to give additional trends when available. The development of validated modelling of the interface between a wheel and the transfer from switch to stock rails will help to reduce the need for continued collection of data that is available and could be developed to continue the trends for deterioration rates.

\section{Acknowledgements}

The project would like to thank Network Rail for their contributions; funding, knowledge and ability to attend and use an operational railway. Contributions from Elias Kassa should also be noted for his initial support to the research.

\section{Nomenclature and Abbreviations}

DOE Design of Experimentation

FEA Finite Element Analysis 


\section{References}

1. Rail Accident Investigation Branch, Rail Accident Report: Derailment at Grayrigg, 23 February 2007: Rail Accident Investigation Branch, Report No: 20/2008, Department for Transport The Wharf, Stores Road, Derby, DE21 4BA. 2009.

2. Network Rail, Annual Return 2013, A technology-enabled future, Network Rail Kings Place, 90 York Way, London, N1 9AG. 2013.

3. Zwanenburg WJ. Modelling Degradation Processes of Switches \& Crossings for Maintenance \& Renewal Planning on the Swiss Railway Network. PhD Thesis: École Polytechnique Federale De Lausanna; 2009.

4. Fox F, River, road and rail - Some engineering reminiscences. London: John Murray; 1904.

5. Cheesewright PR, Wheel/Rail Forces Measured at Crossings of Cast Manganese and Built-up Types, British Railways Research and Development Division - Track Group, June, Report No: 263-191-24, British Rail. 1977.

6. Troup R. M., Measured Wheel-Rail Forces through switch diamonds and swing nose crossings, British Rail Research Board (BRRB), Report No: TM VDY 003, 1985.

7. Boutle NF, A Fatigue-Orientated Analysis of Strains Measured from Two Crossings of Different Design Installed at Watford Junction: British Railways Board, British Railways Research and Development Division - Track Group, Report No: 263-190-16, 1978.

8. Groom GM, Strain Gauge Survey of the E.C.M.L. Newark High Speed Square Crossing, British Railways Research (BRR) - Track Group, Report No: 263-202-9, 1973.

9. Kassa E \& Nielsen JCO, Dynamic interaction between train and railway turnout: full-scale field test and validation of simulation models, Vehicle System Dynamics - International Journal of Vehicle Mechanics and Mobility, 2008; 46(1): 521-534.

10. Kassa E, Andersson C \& Nielsen JCO, Simulation of dynamic interaction between train and railway turnout, Vehicle System Dynamics - International Journal of Vehicle Mechanics and Mobility, 2006; 44(3): 247-258.

11. Pålsson BA \& Nielsen JCO, Wheel-rail interaction and damage in switches and crossings, Vehicle System Dynamics - International Journal of Vehicle Mechanics and Mobility, 2011; 50(1): pp.43-58.

12. Nicklisch, Dirk; Nielsen, Jens C. O.; Ekh, Magnus; Johansson, Anders; Pålsson, Björn; Zoll, Andreas; Reinecke, Jens: Simulation of wheel-rail contact and subsequent material degradation in switches \& crossings. Proceedings 21st International Symposium on Dynamics of Vehicles on Roads and Tracks, Stockholm, Sweden, August 17-21, 2009, 14 pages

13. Williams J, Gordon D \& Harrison J, S\&C Failure and Delay Analysis, Report No: SA/TSS/17754/S3/R001, Serco Assurance Thornson House, Birchwood Park, Warrington, Cheshire, WA3 6GA. 2007.

14. Network Rail. NR/L2/TRK/2049, Track Design Handbook, 40 Melton Street, London, NW1 2EE. Network Rail; 2008.

15. Wickens, A. Fundamentals of Rail Vehicle Dynamics, Guidance and Stability, University of Loughborough, Swets \& Zeitlinger Publishers, 2005. 
16. Cornish A, Kassa E \& Smith RA. Investigation of failure statistics for switches and crossings in the UK. In: Railway Engineering - 2011, Forde M. C. (eds.), University of Westminster, 2011, $1-14$

17. Ventry D, RE/PW/729, BS113A / CEN56E1 Verical switches general arrangement AV-EV, Network Rail, 40 Melton Street, London, NW1 2EE, 2005.

18. Zerbst U, Lunden R, Edel KO \& Smith RA, Introduction to the damage tolerance behaviour of railway rails - a review, Engineering Fracture Mechanics, 2009; 76(17): 2563-2601.

19. Cornish A. Life-time monitoring of in service switches and crossings through field instrumentation. PhD Thesis: Imperial College London, 2014.

20. Jenkins HH, Stephenson JE, Clayton EA, Morland GW \& Lyon D, The Effect of Track and Vehicle Parameters on Wheel/Rail Vertical Dynamic Forces, The Railway Engineering Journal, Institute of Mechanical Engineers, 1974, January; 3(1).

21. Priest J \& Powrie W, Determination of dynamic track modulus from measurement of track velocity during train passage, Journal of Geotechnical and Geoenvironmental Engineering, 2009, November: pp1-33.

22. Le Pen L. Research Fellow, University of Southampton. Personal communication. 26/02/2013.

23. Brucekitchener, Photo 91111, scot-rail.co.uk Scotland's online railway community, [Online] Available from: http://www.scot-rail.co.uk/photo/scaled/1721 [Accessed 12th December 2014] 